\title{
Management of Humeral Shaft Fractures; Non-Operative Versus Operative
}

\author{
Nicholas D. Clement ${ }^{1, *}$ \\ ${ }^{1}$ Department of Orthopedics and Trauma, University of Edinburgh, Edinburgh, UK \\ ${ }^{*}$ Corresponding author: Nicholas D. Clement, Department of Orthopedics and Trauma, University of Edinburgh, Edinburgh, UK. Tel: $+44-1312423497$, Fax: $+44-1312423541$, \\ E-mail: nickclement@doctors.org.uk
}

Received: February 16, 2015; Accepted: March 25, 2015

\begin{abstract}
Context: Functional humeral bracing remains the gold standard for treatment of humeral shaft fractures. There is an increasing trend in the literature to perform operative fixation of these fractures.

Evidence Acquisition: The aim of this systematic review was to compare the level one evidence for the outcome of non-operative with operative management of humeral shaft fractures in adults. A comprehensive electronic literature search of Medline and PubMed was performed with specific inclusion criteria to identify randomized controlled trials.

Results: In total, seventeen different studies were identified from the search terms and combinations used. Only one study met the inclusion criteria; however, this was a published study protocol of an ongoing trial currently being conducted. One additional published protocol for an ongoing trial was also identified, but this was for a prospective comparative observational study. Although this latter study may not be level one evidence, it would offer great insight into the functional outcome of humeral shaft fractures and economic implications of operative management, which is currently not addressed in the literature. Two retrospective comparative studies were also identified, one of which demonstrated a significantly lower rate of nonunion and malunion in those patients undergoing operative management.

Conclusions:This systematic review demonstrated a deficiencyin the current literatureof level one evidence available for themanagement of humeral shaft fractures. The current ongoing randomized control trail would offer a greater insight into the management of humeral shaft fractures and help confirm or refute the current literature. If this randomized control trial affirms the reduction in the rate of nonunion with operative fixation, a cost economic analysis is essential. As it would seem to offer operative management to all patients may be over treatment and not to offer this at all would undertreat.
\end{abstract}

Keywords: Humeral Shaft Fracture; Review; Disease Management

\section{Context}

Humeral shaft fractures are a relatively common fracture presenting to trauma services, with an incidence of 13 per 100000 per year (1). The incidence is dependent upon age and gender, with an overall bimodal distribution due to a peak incidence for males between 20 to 30 years old and a second peak for older females aged between 60 and 70 years (2). It is thought that the incidence may increase many fold in the future due to the growing elderly population, which would have significant repercussions on health care services (3). Management of these fractures would form a growing part of trauma management in the future and an understanding of the current literature and deficiencies in evidence is essential to optimize the outcome of patients.

Non-operative management of humeral shaft fractures using functional bracing is the currently accepted gold standard of treatment (4). The Egyptians described managing humeral shaft fracture with splints over 3500 years ago, which remained the established treatment method until more recently (4). Splints and casts generally included the elbow and shoulder which would often result in subsequent stiffness to the joint once the treatment regimen was complete. Sarmiento et al. (5) recognized this morbidity and described the outcome of functional bracing for the treatment of humeral shaft fractures. This functional brace is applied to the arm once acute pain and swelling subside and is recommended as soon as possible, once the patient can tolerate it. Application would normally be between a week to two weeks following the fracture. The functional brace is a prefabricated polypropylene sleeve, which is fitted to the patient to encompass the arm and allow compression of the soft tissues using the adjustable Velcro straps and does not inhibit shoulder or elbow movement (Figure 1). This compression is thought to help immobilize the fracture site and enable early mobilization of the shoulder and elbow preventing secondary stiffness (5).

Functional bracing of humeral shaft fractures may be the current gold standard treatment; however, more recent studies questioned this management for all fractures $(6,7)$. Denard et al. (6) described a retrospective comparative study of operative versus non-operative

Copyright (C) 2015, Kashan University of Medical Sciences. This is an open-access article distributed under the terms of the Creative Commons Attribution-NonCommercial 4.0 International License (http://creativecommons.org/licenses/by-nc/4.0/) which permits copy and redistribute the material just in noncommercial usages, provided the original work is properly cited. 


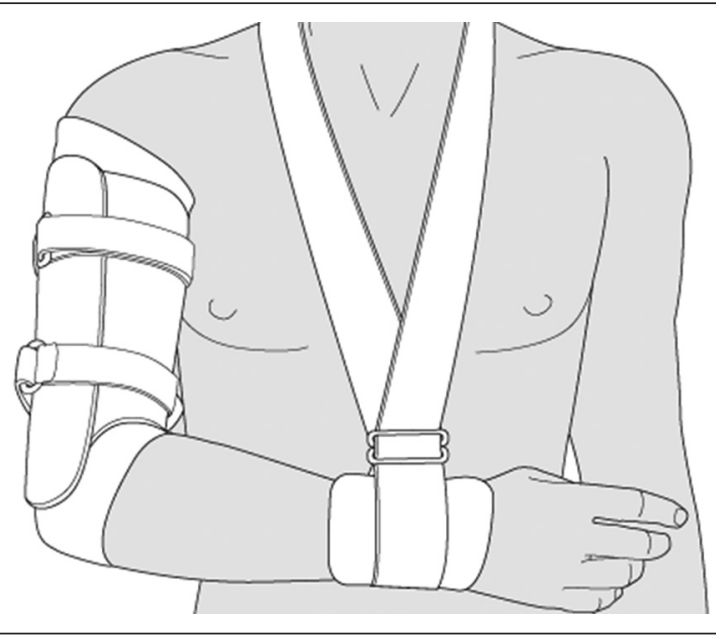

Figure 1. A Diagram of a Functional Humeral Brace

management of humeral shaft fractures. They observed a significantly lower rate of nonunion and malunion in those patients undergoing operative management, although they did not find any difference in the final range of motion between these groups. Therefore, authors concluded that "in certain clinical scenarios, these fractures may be well severed by compression plating" (6). More recently, a review of non-operative management for humeral shaft fractures demonstrated a higher rate of nonunion for proximal third fractures and suggested that a lower threshold of surgical fixation of such fractures may be considered (7). This trend in the current literature towards operative fixation of selected humeral shaft fractures is reflected in a study from Finland where the rate of surgical treatment was demonstrated to have doubled over the last two decades (8).

Open reduction and plate fixation (Figure 2) and intramedullary nailing (Figure 3 ) are the two most commonly recognized operative methods of fixation for humeral shaft fractures. There have been multiple randomized controlled trails comparing the outcome of these methods of fixation; however, there are some advantages and disadvantages for both techniques (9). A meta-analysis of five studies including 237 patients demonstrated that a lower complication rate was observed with open reduction and plate fixation (10).

The aim of this systematic review was to compare the outcome of non-operative and operative management of humeral shaft fractures in adults.

\section{Evidence Acquisition}

The inclusion criteria for this review were all randomized controlled trials comparing the outcome of nonoperative versus operative management of non-pathological acute humeral shaft fractures in adults (16 years and older). Non-operative management was defined to include: sugar tong and plaster splints, hanging casts or functional humeral bracing. Operative interventions

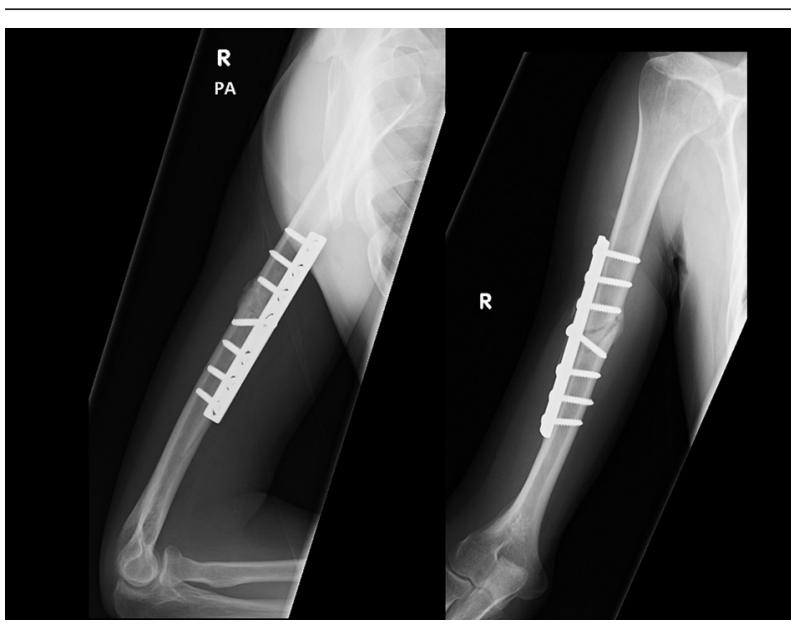

Figure 2. A Radiograph Obtained Six Months After Primary Open Reduction and Compression Plate Fixation of a Midshaft Humeral Fracture

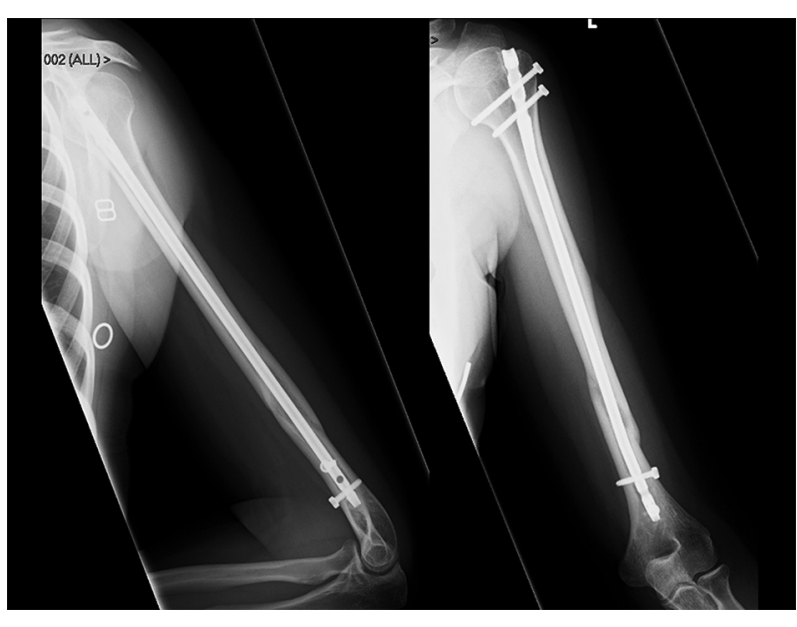

Figure 3. A Radiograph Obtained Nine Months After Primary Intramedullary Nailing of a Segmental Humeral Fracture

were defined to include intramedullary nailing, external fixation and open reduction and plate fixation. Studies comparing different methods of non-operative treatment alone or different methods of operative management alone were excluded.

Ovid Medline (1946 to the $13^{\text {th }}$ of February 2015) and PubMed were used as electronic search engines for this review. The search terms and combinations used to identify the studies from Medline are included in Table1. Two search terms were used in PubMed: "((((humeral) AND fracture) AND shaft) and randomized) and operative )" and "(((()humeral) and fracture) and shaft) and randomized) and operative)", which identified three and seven studies, respectively. All studies were then securitized to assess their eligibility for inclusion according to the defined criteria.

\section{Results}

In total, seventeen different studies were identified from the search terms and combinations used. Only 
Table 1. Medline Search Terms and Combinations to Identify Studies That Achieved the Inclusion Criteria

\begin{tabular}{lc}
\hline Search Terms $(\mathbf{n})$ & Combinations $(\mathbf{n})$ \\
\hline 1. Humeral $(\mathbf{1 2 4 2 7})$ & 1 or 2(21156) \\
\hline 2. Humerus $(\mathbf{1 4 5 7 4})$ & 4 or $5(16397)$ \\
\hline 3. Fracture $(\mathbf{1 2 9 6 1 4})$ & 6 or $7(572147)$ \\
\hline 4. Shaft $(\mathbf{1 2 7 8 3 )}$ & 11 and 8 and 13 and $14(10)$ \\
\hline 5. Diaphysis $(\mathbf{3 9 8 3})$ & \\
\hline 6. Randomized $(\mathbf{6 0 3 3 4})$ & \\
\hline 7. Randomized $(\mathbf{5 5 7 0 9 1})$ & \\
\hline
\end{tabular}

one study met the inclusion criteria; however, this was a published study protocol of an ongoing trial currently being conducted (11). One additional published protocol for an ongoing another trial was also identified, but this was a prospective comparative observational study (12). Although this latter study may not be level one evidence, it would offer great insight into the functional outcome of humeral shaft fractures and cost economic implications of operative management, which is currently not addressed in the literature.

\section{Conclusions}

This systematic review failed to identify any randomized controlled trials comparing the outcome of nonoperative with operative management of humeral shaft fractures. The only current evidence for the management of these fractures is in the form of retrospective comparative studies. However, this void in the literature is currently being addressed by an ongoing randomized controlled trial that would hopefully answer which method of treatment of humeral shaft fractures results in a superior outcome for patient. The remainder of this review would critically discuss the current retrospective studies published comparing the outcome of operative with non-operative management of humeral shaft fractures. In addition, the current ongoing randomized (11) controlled trial and the prospective comparative observational study (12) identified in this review would be discussed.

Denard et al.(6) recognized the deficiency in the current literature of comparing the outcome of non-operative and operative management of humeral shaft fractures. They retrospectively identified two-hundred thirteen adult patients presenting to two level one trauma centers with a humeral shaft fracture and managed with either a functional brace (being defined as the non-operative treatment group) or compression plating (being defined as the operative treatment group). The primary outcome measures assessed were time to union, nonunion, malunion, infection, incidence of radial nerve palsy and elbow range of motion. They observed the rate of nonunion in operative group (9\%) to be less than a half of that in non-operative group (21\%), which was statistically significant. The rate of malunion was also significantly reduced in the operative group (1\% versus $13 \%$ ). There was however no significant difference in the rate of infection, radial nerve palsy, time to union or range of motion between the two groups. Hence the authors concluded that operative management had a significantly lower rate of nonunion and malunion with no observed differences in time to union, infection or iatrogenic radial nerve palsy. There are major limitations of this selected retrospective comparative study, which may explain the differing rate of nonunion demonstrated between the groups with patients who may not be such good surgical candidates not being offered surgery. The declared demographics and morbidity of the two groups were comparable, and there was a greater rate of smokers in the operative group that may have predisposed them to a greater rate of nonunion (13). This is the best comparative study to date and although there are major limitations, it certainly supports operative fixation of humeral shaft fractures in specific clinical scenarios.

A more recent retrospective comparative study by Mahabier et al. (14) affirmed the equal complication rates between non-operative and operative management of humeral shaft fractures as observed by Denard et al. (6). Mahabier et al. (14) retrospectively identified 186 patients aged 16 years or more presented to the study center with a humeral shaft fracture during a 5-year period. Ninetyone patients were treated non-operatively and 95 were treated operatively. The rate of radial nerve palsy was similar between the non-operative (9\%) and operative groups (10\%); however, the authors stated that $5 \%$ of patients incurred their radial nerve palsy as a direct result of operation. Unusually the authors did not observe any nonunions in their series, but described 36 patients with a delayed union defined as failure to heal at 24 weeks post fracture with no progression to healing. The delayed union rates between the two groups were identical (19\%). In contrast to the aforementioned study of Denard et al. (6), most patients in this study underwent intramedullary nailing of the humerus ( $n=78 / 95)$, and this may explain the equivocal rate of union between non-operative and operative groups (10).

If it is assumed from the results of these two comparative studies that operative fixation, with compression plating, offers a lower rate of nonunion and malunion without a significant increase in the complication rate, the question has to be asked whether this is beneficial to patient. The rate of nonunion of humeral shaft fractures varies in the literature from 0 to $23 \%(5,15)$. There is general agreement in the literature that a good to excellent outcome can be achieved with operative fixation of a nonunion with a union rate of approximately $90 \%(4,16)$. Although these results seem acceptable, a patient would endure three to six months or even longer of symptoms from their nonunion before undergoing surgery. Hence, it would not seem correct to wait until a nonunion is es- 
tablished, but to offer operative fixation for all patients would be over treatment and to offer fixation to no patients would seem to be undertreating. Patients at high risk of a nonunion, such as those with more proximal humeral shaft fractures or poor early functional improvement $(7,17)$, may benefit most from operative fixation. For example, if the overall nonunion rate is $17 \%$ (17), the number of needed operations to prevent one nonunion would be six; however, this would decrease to potentially a half the number if only those patients at risk of nonunion offered fixation.

Current reviews would suggest that a malunion of less than 20 degrees in sagittal plain and less than 30 degrees in coronal plane with shortening of less than 2 to $3 \mathrm{~cm}$ are considered acceptable (Figure 4$)(4,18)$. This is however based on a single study from 1966 that only assessed the outcome of 32 patients with no validated outcome assessment or patient reported outcome measure (19) The reported rate of malunion by Denard et al. (6) of 13\% in the non-operative group is similar to that reported by Rutgers and Ring (20) of $9 \%$ in their series of 52 nonoperatively managed patients. We are unaware of a published study that assessed the correlation of functional outcome with radiographic deformity; it would seem intuitive that with worsening deformity, function may be deteriorated. If this is the case, then the reduction in the rate of malunion to $1 \%$, as described by Denard et al.(6), in the operative group should be recognized with a better functional outcome. This is an essential aspect of future studies assessing the outcome of humeral shaft fractures.

This systematic review identified two ongoing trails currently being performed in Brazil (11) and the Netherlands (12) to further clarify the outcome of humeral shaft fractures. Matsunaga et al. (11) published their protocol for a two arm randomized controlled trial comparing the outcome of bridge plating with functional bracing. They proposed to recruit 110 patients with humeral shaft fractures. A minimally invasive technique, as described by

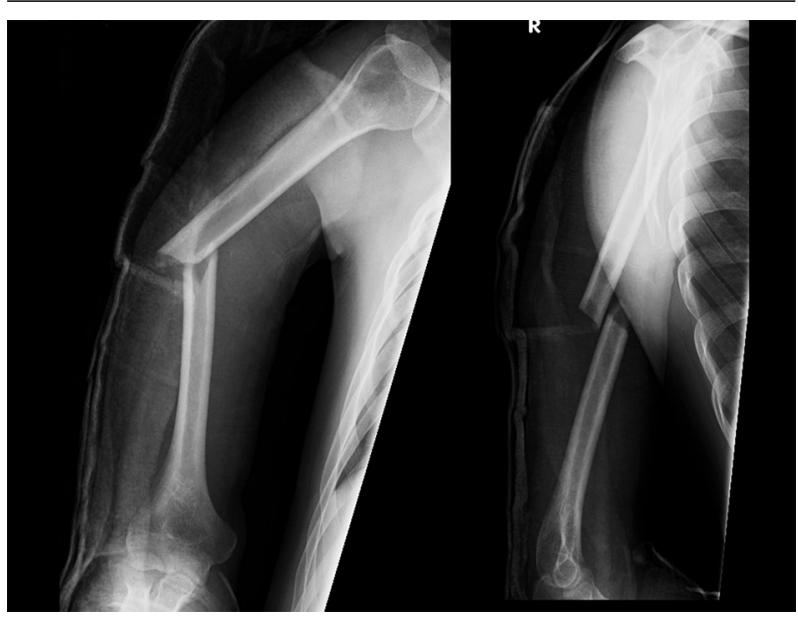

Figure 4. A Radiograph of a Patient One Week Following the Humera Midshaft Fracture Demonstrating a Perceived Acceptable Position
Livani and Belangero (21), using a narrow dynamic compression plate is to be used in the operative arm of the study and would be compared to a functional brace in non-operative arm of the trial. The rehabilitation protocol would be standardized for all patients followed up for one year. Their primary outcome is disability of the arm, shoulder and hand score at six months, and their secondary outcomes include Short Form 36 questionnaire, complications, Constant score, subjective pain (Visual Analogue Scale) and radiographic assessment.

Mahabier et al. (12) performed a prospective multicenter observational study on 400 patients with sustained humeral shaft fracture. The management decision, as to whether this is non-operative or operative would be left to the discretion of the treating surgeon. Similarly, their primary outcome measure is disabilities of the arm, shoulder and hand score, which would be assessed at regular intervals over a follow-up period of one year. Secondary outcome measures are the Constant score, subjective pain assessment, range of motion, radiographic union, complications, health-related quality of life using the Short-Form 36 and EuroQol-5D, and time to recommencement of activities of daily living and work and cost-effectiveness. Due to the large data set, the authors proposed to use multivariable regression analyses to adjust confounding variables when identifying independent predictors of outcome. Despite this, not randomizing patients to one treatment method or another would be a major limitation of this study. The main strength of this study would be the cost-effectiveness analysis, which is essential to assess whether the outcome of operative management is superior to non-operative management to justify such an intervention.

This systematic review demonstrated a deficiency in the current literature of level one evidence available for the management of humeral shaft fractures. The available comparative data is limited, but there seems to be an equal complication rate associated with non-operative and operative management with the advantage of a lower rate of nonunion and malunion observed in patients undergoing operative management. However, to potentially operate on six patients to prevent one nonunion may not be the best use of the available resources. This ratio could be reduced if those at greatest risk of nonunion, such as proximal fractures, were offered surgery primarily. The current ongoing randomized control trail would offer a greater insight into the management of humeral shaft fractures and help confirm or refute the current literature. If this randomized control trial affirms reduction in the rate of nonunion with operative fixation, a cost economic analysis is essential. As it seems that offering operative management to all patients may be over treatment and not to offer this at all would undertreat. Hence, there must be a middle ground of offering surgery to patients with predicators of nonunion or a poor outcome and this should be addressed in future studies. 


\section{Authors' Contributions}

Nicholas D. Clement conceived the idea for the review, performed the literature review, composed the manuscript and submitted the final manuscript.

\section{References}

1. Court-Brown CM, Caesar B. Epidemiology of adult fractures: A review. Injury. 2006;37(8):691-7.

2. Tytherleigh-Strong G, Walls N, McQueen MM. The epidemiology of humeral shaft fractures. J Bone Joint Surg Br.1998;80(2):249-53.

3. Kim SH, Szabo RM, Marder RA. Epidemiology of humerus fractures in the United States: nationwide emergency department sample, 2008. Arthritis Care Res (Hoboken). 2012;64(3):407-14.

4. Walker M, Palumbo B, Badman B, Brooks J, Van Gelderen J, Mighell M. Humeral shaft fractures: a review. J Shoulder Elbow Surg. 2011;20(5):833-44.

5. Sarmiento A, Kinman PB, Galvin EG, Schmitt RH, Phillips JG. Functional bracing of fractures of the shaft of the humerus. $J$ Bone Joint Surg Am. 1977;59(5):596-601.

6. Denard AJ, Richards JE, Obremskey WT, Tucker MC, Floyd M Herzog GA. Outcome of nonoperative vs operative treatment of humeral shaft fractures: a retrospective study of 213 patients. Orthopedics. 2010;33(8)

7. Ali E, Griffiths D, Obi N, Tytherleigh-Strong G, Van Rensburg L. Nonoperative treatment of humeral shaft fractures revisited. $J$ Shoulder Elbow Surg. 2015;24(2):210-4.

8. Huttunen TT, Kannus P, Lepola V, Pihlajamaki H, Mattila VM. Surgical treatment of humeral-shaft fractures: a register-based study in Finland between 1987 and 2009. Injury. 2012;43(10):1704-8.

9. Vennettilli M, Petrisor B, Athwal GS. Operative treatment of diaphyseal humeral fractures. J Hand Surg Am. 2011;36(5):905-6.

10. Heineman DJ, Bhandari M, Nork SE, Ponsen KJ, Poolman RW.
Treatment of humeral shaft fractures--meta-analysis reupdated. Acta Orthop. 2010;81(4):517.

11. Matsunaga FT, Tamaoki MJ, Matsumoto MH, dos Santos JB, Faloppa F, Belloti JC. Treatment of the humeral shaft fractures-minimally invasive osteosynthesis with bridge plate versus conservative treatment with functional brace: study protocol for a randomised controlled trial. Trials. 2013;14:246

12. Mahabier KC, Van Lieshout EM, Bolhuis HW, Bos PK, Bronkhorst MW, Bruijninckx MM, et al. HUMeral shaft fractures: measuring recovery after operative versus non-operative treatment (HUMMER): a multicenter comparative observational study. BMC Musculoskelet Disord. 2014;15:39.

13. Ding L, He Z, Xiao H, Chai L, Xue F. Factors affecting the incidence of aseptic nonunion after surgical fixation of humeral diaphyseal fracture. J Orthop Sci. 2014;19(6):973-7.

14. Mahabier KC, Vogels LM, Punt BJ, Roukema GR, Patka P, Van Lieshout EM. Humeral shaft fractures: retrospective results of non-operative and operative treatment of 186 patients. Injury. 2013;44(4):427-30.

15. Toivanen JA, Nieminen J, Laine HJ, Honkonen SE, Jarvinen MJ Functional treatment of closed humeral shaft fractures. Int Orthop. 2005;29(1):10-3.

16. Wright TW. Treatment of humeral diaphyseal nonunions in patients with severely compromised bone. J South Orthop Assoc. 1997;6(1):1-7.

17. Broadbent MR, Will E, McQueen MM. Prediction of outcome after humeral diaphyseal fracture. Injury. 2010;41(6):572-7.

18. Carroll EA, Schweppe M, Langfitt M, Miller AN, Halvorson JJ. Management of humeral shaft fractures. J Am Acad Orthop Surg. 2012;20(7):423-33.

19. Klenerman L. Fractures of the shaft of the humerus.J Bone Joint Surg Br.1966;48(1):105-11.

20. Rutgers M, Ring D. Treatment of diaphyseal fractures of the humerus using a functional brace.J Orthop Trauma. 2006;20(9):597-601.

21. Livani B, Belangero WD. Bridging plate osteosynthesis of humeral shaft fractures. Injury. 2004;35(6):587-95. 